\title{
Dual spacecraft determinations of magnetopause motion
}

\author{
T. M. Bauer ${ }^{1}$, M. W. Dunlop ${ }^{2}$, B. U. Ö Sonnerup ${ }^{3}$, N. Sckopke ${ }^{1,5}$, \\ A. N. Fazakerley ${ }^{4}$, and A. V. Khrabrov ${ }^{3}$
}

\begin{abstract}
We examine the motion of Earth's magnetopause for 16 dawnside traversals of this boundary by the sister spacecraft AMPTE/UKS and IRM in December, 1984, when their separation was $400-900 \mathrm{~km}$. We compare magnetopause normal vectors, $\mathrm{n}$, and speeds of motion, $u_{n}$, obtained separately from each spacecraft by use of three different methods, and also compare those $u_{n}$ to corresponding speeds, $u_{n}^{*}$, obtained from observed time lags between the two spacecraft. Agreement between $u_{n}$ values and $n$ vectors determined from the three methods ranges from poor to excellent. Comparing $u_{n}^{*}$ and $u_{n}$ values, we find a clear tendency for $\left|u_{n}^{*}\right|$ to be larger than $\left|u_{n}\right|$ : While slightly less than half of the results show reasonable agreement $\left(0.5<u_{n} / u_{n}^{*}<2\right)$, there are about as many results in the range $0<u_{n} / u_{n}^{*}<0.5$, and a few cases give the wrong sign of $u_{n}$.
\end{abstract}

\section{Introduction}

That Earth's magnetopause is continually moving inward and outward, sometimes at large velocities, became evident immediately following the first extensive in-situ observations of this electric current layer [Cahill and Amazeen, 1963]. Statistical information about its speed, $u_{n}$, was derived early on by a variety of methods and yielded typical values in the range $10-60 \mathrm{~km} / \mathrm{s}$. Kaufmann and Konradi, [1969] then pioneered a remote sensing technique, based on measurements of energetic ions, that yielded the first direct determinations of $u_{n}$ from single-spacecraft data. They found velocities that were usually less than $20 \mathrm{~km} / \mathrm{s}$ but occasionally large, with a maximum value of $156 \mathrm{~km} / \mathrm{s}$ derived for one case. Much later, $u_{n}$ and its statistical spread were determined directly from time-lag measurements by the spacecraft pair ISEE 1 and 2 [Berchem and Russell, 1982]. Speeds in the range 5$380 \mathrm{~km} / \mathrm{s}$ were found, with $80 \%$ of the events falling in the range $10-80 \mathrm{~km} / \mathrm{s}$.

The possibility of using magnetic field (B) and plasma velocity ( $v$ ) measurements from a single spacecraft to determine $u_{n}$ started to be explored in the eighties, using the de Hoffmann-Teller (HT) frame velocity. This velocity, $\mathbf{V}_{\mathrm{HT}}$, is obtained by minimizing the average $\left\langle\left(\mathbf{v}^{k}-\right.\right.$ $\left.\left.\mathbf{V}_{\mathrm{HT}}\right) \times\left.\mathbf{B}^{k}\right|^{2}\right\rangle$ over all $M$ individual data pairs $\left\{\mathbf{v}^{k}, \mathbf{B}^{k}\right\}$, $k=1,2, \ldots M$, measured during a magnetopause crossing [Sonnerup et al., 1987]. The HT frame is the "proper" frame of magnetic structures carried by the plasma flow

\footnotetext{
${ }^{1}$ MPI für extraterrestrische Physik, Garching, Germany

2Imperial College, London, UK

${ }^{3}$ Dartmouth College, Hanover, NH, USA

${ }^{4}$ Mullard Space Science Lab, Surrey, UK

${ }^{5}$ Deceased November 28, 1999
}

Copyright 2000 by the American Geophysical Union.

Paper number 2000GL000041.

0094-8276/00/2000GL000041\$05.00 past the observing spacecraft. Its velocity along the magnetopause normal, $\mathbf{n}$, therefore represents the motion of this current layer past the spacecraft, i.e., $u_{n}=\mathbf{V}_{\mathrm{HT}} \cdot \mathbf{n}$. The vector $\mathbf{n}$ can be obtained from measured $\mathbf{B}$ vectors by minimization of the variance of the field component along $\mathbf{n}$ [e.g., Sonnerup and Scheible, 1998], a technique referred to as MVB. For brevity, we denote the combined MVB and HT analysis as MVBHT.

More recently, Terasawa et al., [1996] developed a method whereby $n$ and $u_{n}$ can be determined together in a single minimization of the variance of the electric field tangential to the magnetopause, which for a strictly one-dimensional structure moving at constant speed should be zero, in accordance with Faraday's law [see also Khrabrov and Sonnerup, 1998]. This method is called Minimum Faraday Residue (MFR) analysis.

Still another technique, referred to as MVBTD, is based on the assumption that the magnetopause can be approximately described as a tangential discontinuity (TD). In this approach, $\mathbf{n}$ is determined from MVB with the added constraint $\left\langle\mathbf{B}^{k}\right\rangle \cdot \mathbf{n}=\mathbf{0}$ [e.g., Sonnerup and Scheible, 1998]; $u_{n}$ is then obtained as $\left\langle\mathbf{v}^{k}\right\rangle \cdot \mathbf{n}$. The MVBTD technique can also be applied for rotational discontinuities if the reconnection plasma flow across the magnetopause is small compared to the speed of the magnetopause [Phan and Paschmann, 1996].

The magnetopause study presented here provides an opportunity to compare results from the MVBHT, MFR, and MVBTD techniques and to compare results concerning $\mathbf{n}$ and $u_{n}$ obtained from two observation platforms located some $400-900 \mathrm{~km}$ apart. It also permits comparison of $u_{n}$ with velocities $u_{n}^{*}=\Delta \mathbf{R} \cdot \mathbf{n} / \Delta t$, obtained from the time lag $\Delta t=\left(t_{\mathrm{UKS}}-t_{\mathrm{IRM}}\right)$ between passages of the magnetopause over the two spacecraft. Here $\Delta \mathbf{R}=\left(\mathbf{R}_{\text {UKS }}-\mathbf{R}_{\text {IRM }}\right)$ is the spacecraft separation vector. The determination of $\Delta t$ is often made difficult by the presence of localized tempo$\mathrm{ral} / \mathrm{spatial}$ variations within the magnetopause. We choose time lags $\Delta t$ that give high (maximum) cross correlation between UKS and IRM magnetic data and that are consistent with the passage of any pronouced substructures across the two spacecraft. If the passage of substructures rather than the entire magnetopause is used for determination of $\Delta t$ then $u_{n}^{*}$ represents the magnetopause velocity only if the substructures are planar with their normal along $\mathbf{n}$. The true sign of the magnetopause speed is always unambiguously known, since the direction of the traversal - from magnetosheath to magnetosphere $\left(u_{n}>0\right)$ or vice versa $\left(u_{n}<0\right)-$ is known from the time series.

Events for our study were selected by use of two constraints: (1) plasma and $B$ data must be available from both UKS and IRM; (2) the spacecraft separation must be large $(\gg 40 \mathrm{~km})$ to avoid having existing uncertainties in $\Delta \mathbf{R}$ compromise the determination of $u_{n}^{*}$. 


\section{Crossing on December 11, 1984}

To illustrate the procedure and results, we now examine a single magnetopause crossing on Dec. 11, 1984, in detail. On this occasion, the spacecraft were on the inbound leg of their orbit, with IRM leading UKS by $720 \mathrm{~km}$. The magnetopause encounter occurred near the GSE equatorial plane at 9:40 local time at a distance of $9 R_{E}$ from Earth. The magnetopause travelled outward $\left(u_{n}>0\right)$ past the two spacecraft, i.e., a transition from magnetosheath to magnetosphere conditions was observed. Time plots of field magnitude $|\mathbf{B}|$ are shown in Figure 1. Optimum intervals for determination of $\mathrm{n}$ and $u_{n}$ are shown by heavy horizontal bars.

The two most pronounced features in Figure 1, the $|B|$ minima denoted by "Dip 1" and "Dip 2", were used for determination of an average $\Delta t$ value of $48 \mathrm{~s}$. Results for $u_{n}$ and $u_{n}^{*}$ are given in Table 1 . The six $u_{n}^{*}$ values differ slightly from each other, because the six $n$ vectors used to obtain $u_{n}^{*}$ are slightly different. In an overall sense, the agreement between $u_{n}$ from the three single-spacecraft techniques is good for this event. The agreement with corresponding $u_{n}^{*}$ values is also good.

The $\mathbf{n}$ vectors and their error ellipses are presented in Figure 2. It is seen that the (1- $\sigma$ ) error ellipses, which represent statistical but not systematic errors, are relatively small and overlap only partly. The UKS vectors agree with each other within about $2.5^{\circ}$ and the IRM vectors within about $4^{\circ}$. But the IRM vectors are rotated away from the UKS vectors by some $6-8^{\circ}$, representing perhaps a temporal change of $\mathbf{n}$.

If the normals from MVBTD are multiplied by $V_{H T}$ rather than by $\left\langle v^{k}\right\rangle$, the resulting $u_{n}$ values (UKS $10.0 \mathrm{~km} / \mathrm{s}$, IRM $7.0 \mathrm{~km} / \mathrm{s}$ ) remain very close to those obtained for $\left\langle v^{k}\right\rangle$ and given in Table 1 . No plasma acceleration was observed. We conclude that the magnetopause was a tangential discontinuity with purely tangential plasma flow and no reconnection but note that the MVB and MFR normal vectors did not yield $B_{n}=\left\langle\mathbf{B}^{k}\right\rangle \cdot \mathbf{n}$ precisely equal to zero (UKS $2.0 \pm 1.8 \mathrm{nT}$ and IRM $5.3 \pm 2.1 \mathrm{nT}$ from MVB normal; UKS $0.5 \pm 2.0 \mathrm{nT}$ and IRM $3.5 \pm 4.0 \mathrm{nT}$ from MFR normal).

\section{Additional Events}

We have also examined three magnetopause passes on Dec. 19, Dec. 21, and Dec. 22, 1984. For each of these passes we identified five individual crossings of the magnetopause and analyzed them in the same way as the Dec. 11 event.

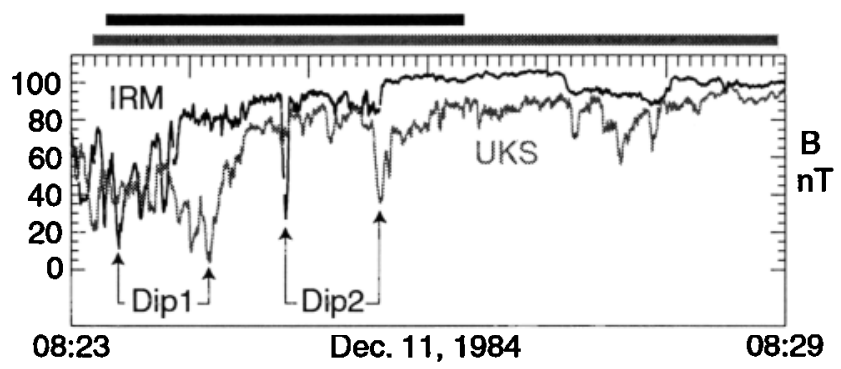

Figure 1. Time series of field magnitude $|B|$ on Dec. 11, 1984, with UKS data shown by light curves and IRM by dark curves. Horizontal bars represent time intervals used for the calculations. Dip 1 and Dip 2 were used for time-lag estimate of magnetopause velocity.
Table 1. Magnetopause speeds on Dec. 11 in $\mathrm{km} / \mathrm{s}$

\begin{tabular}{lcccccc}
\hline \multirow{2}{*}{ Spacecraft } & \multicolumn{2}{c}{ MVBHT } & \multicolumn{2}{c}{ MFR } & \multicolumn{2}{c}{ MVBTD } \\
\cline { 2 - 7 } & $u_{n}$ & $u_{n}^{*}$ & $u_{n}$ & $u_{n}^{*}$ & $u_{n}$ & $u_{n}^{*}$ \\
\hline UKS & 11.7 & 10.7 & 13.0 & 11.0 & 9.8 & 10.7 \\
IRM & 17.7 & 9.9 & 14.5 & 10.5 & 6.9 & 10.0 \\
\hline
\end{tabular}

All three passes occurred near the GSE equatorial plane. On Dec. 22 the two spacecraft were inbound at a location (8:50 local time, distance $10 R_{E}$ ) similar to the Dec. 11 event. The passes on Dec. 19 and 21 were outbound and occurred farther tailward at 6:00 local time at a distance of $14.5 R_{E}$.

By comparing the $n$ vectors of sequences of crossings within each of the three passes, we find that the normal rotated back and forth by some $10-50^{\circ}$, indicating that the magnetopause was strongly undulated due to surface waves or, perhaps, reconnection pulses. Dividing these $10-50^{\circ}$ changes in normal direction by the time spacing between crossings, one derives that the normals rotated with speeds of typically $0.1^{\circ} / \mathrm{s}$. Multiplying this rotation speed again with the lengths (20-300 s) of our magnetopause analysis intervals, we find that within those intervals the normal direction may have changed by some $2-30^{\circ}$. Under such conditions, there is, strictly speaking, no unique normal. However, the MVB and MFR techniques should find the average orientation within each analysis interval.

For the statistics described below, we accept the $\mathbf{n}$ vectors from MVB or from MFR of a given crossing only if the corresponding ratio of intermediate to minimum eigenvalue $\left(\lambda_{2} / \lambda_{3}\right)$ exceeds 4 . Using this selection criterion, we rejected all results for the third crossing on Dec. 19, all results for the second crossing on Dec. 21, and the MFR result from IRM for the third crossing on Dec. 21. We also eliminated the $u_{n}^{*}$ results from MFR and MVBTD for the second IRM crossing on Dec. 22, because for these $n$ vectors the separation $\Delta \mathbf{R} \cdot \mathbf{n}$ of the spacecraft along $\mathbf{n}$ was less than $50 \mathrm{~km}$ and thus comparable to existing uncertainties in $\Delta \mathbf{R}$.

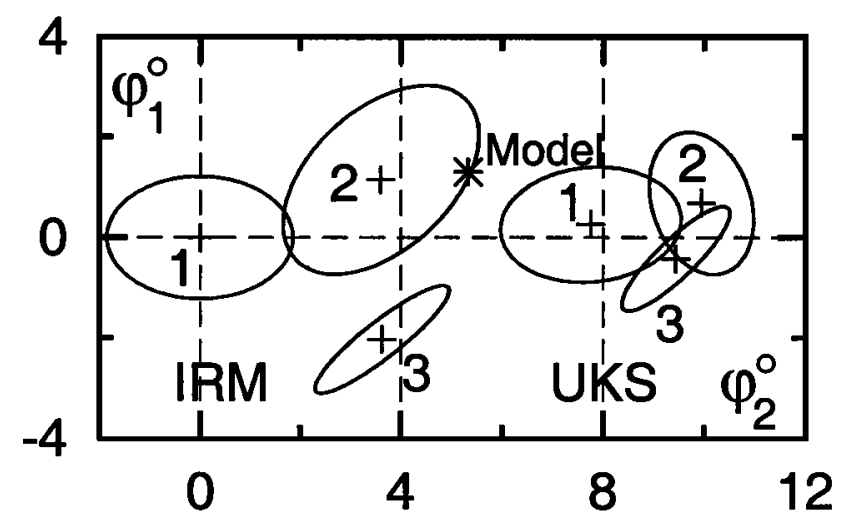

Figure 2. Partial map of unit sphere, showing estimates of normal $n$ and 1- $\sigma$ error ellipses on Dec. 11, 1984, using data from UKS and IRM. Techniques used are: (1) MVB; (2) MFR; (3) MVBTD. Axes indicate angular deviations relative to the MVB normal from IRM. The asterisk marks a model normal [Fairfield, 1971]. 
Table 2. Statistics of angles between normal vectors: $\Lambda_{12}$ is the angle between $\mathbf{n}_{M V B}$ and $\mathbf{n}_{\mathrm{MFR}}, \Lambda_{13}$ between $\mathbf{n}_{\mathrm{MVB}}$ and $\mathbf{n}_{\text {MVBTD }}, \Lambda_{23}$ between $\mathbf{n}_{\text {MFR }}$ and $\mathbf{n}_{\text {MVBTD }} \cdot \Lambda_{11}, \Lambda_{22}, \Lambda_{33}$ are the angles between $\mathbf{n}_{M V B}, \mathbf{n}_{\text {MFR }}, \mathbf{n}_{\text {MVBTD }}$ of UKS and the corresponding IRM normals. Listed is the number of results for which $\Lambda<10^{\circ}, 10^{\circ}<\Lambda<20^{\circ}$, and $\Lambda>20^{\circ}$, respectively.

\begin{tabular}{ccccccc}
\hline Range & $\Lambda_{12}$ & $\Lambda_{13}$ & $\Lambda_{23}$ & $\Lambda_{11}$ & $\Lambda_{22}$ & $\Lambda_{33}$ \\
\hline $0-10^{\circ}$ & 19 & 15 & 15 & 9 & 7 & 10 \\
$10-20^{\circ}$ & 7 & 7 & 5 & 5 & 3 & 2 \\
$20-180^{\circ}$ & 1 & 6 & 7 & 0 & 3 & 2 \\
\hline
\end{tabular}

For the passes on Dec. 19, 21, and 22, in which multiple crossings occurred, the agreement between $\mathbf{n}$ vectors and between magnetopause speeds is, on the whole, less good than on Dec. 11. Table 2 contains information about the angles between the various normal vectors. For each spacecraft, we computed the angle $\Lambda_{12}$ between $n$ from MVB and $n$ from MFR, the angle $\Lambda_{13}$ between $n$ from MVB and $n$ from MVBTD, and the angle $\Lambda_{23}$ between $n$ from MFR and $n$ from MVBTD. Moreover, we computed the angle $\Lambda_{11}$ between the MVB normals from the two spacecraft, the angle $\Lambda_{22}$ between the two MFR normals, and the angle $\Lambda_{33}$ between the two MVBTD normals. It can be seen that these angular deviations often exceed $10^{\circ}$, sometimes even $20^{\circ}$. In view of the $2-30^{\circ}$ changes of the normal direction within an analysis interval, these deviations are not surprising.

The lower scatter plot in Figure 3 compares the values of $u_{n}$, obtained from MVBHT, MFR, and MVBTD, with the corresponding values of $u_{n}^{*}$. Seven data points fall in the second and fourth quadrant of the plot, indicating that the sign of $u_{n}$ was inconsistent with that obtained from $u_{n}^{*}$ (which corresponds to the actual direction of the traversal). Of the 81 data points, 36 fall in the range $0.5<u_{n} / u_{n}^{*}<2$, which may be considered reasonable agreement. The remaining data points show a clear tendency for $\left|u_{n}^{*}\right|$ to come out substantially larger than $\left|u_{n}\right|$ : While only 1 point has $u_{n} / u_{n}^{*}>2$, there are 37 points in the range $0<u_{n} / u_{n}^{*}<0.5$. The upper scatter plot in Figure 3 shows the $u_{n}$ values from MFR and MVBTD versus the $u_{n}$ values from MVBHT for both spacecraft. The slope of the regression line for the combined set of values from MFR and MVBTD is 0.95 , with correlation coefficient 0.94 .

\section{Discussion}

The selection of events for this study was based on availability of data from both spacecraft and on large spacecraft separation. Concerning the quality of the determination of $\mathbf{n}$ from MVB and from MFR, we only required the ratio of intermediate to minimum eigenvalue $\left(\lambda_{2} / \lambda_{3}\right)$ to exceed 4 . A common rule of thumb is that $\lambda_{2} / \lambda_{3}$ should exceed 10 in order for the normal vector determination to be considered reliable. This stricter criterion is met by 16 of the 28 MVB results and by 12 of the 27 MFR results. Restricting our study to this subset would have reduced the angles between $n$ vectors and the differences between the $u_{n}$. For the Dec. 11 event, the $\lambda_{2} / \lambda_{3}$ values range from 14 to 32 .

The sign of $u_{n}$ is found to be correct for 27 of the 28 MVBHT results, for 24 of the 26 MFR results, and for 23 of the 27 MVBTD results. The probability that these ratios are random is very low $\left(10^{-7}\right.$ for $27 / 28,5 \times 10^{-6}$ for $24 / 26,10^{-4}$ for 23/27). As indicated in Figure 3, the agreement between $u_{n}$ obtained from the three methods ranges from excellent to poor. We illustrate this further by comparing $\bar{u}_{n}$, the average of the six (in few cases five) $u_{n}$ values calculated for each magnetopause crossing with the standard deviation $\Delta u_{n}$ of the $u_{n}$ values entering $\bar{u}_{n}$. For 5 of the 14 crossings $\Delta u_{n} /\left|\bar{u}_{n}\right|$ is below $1 / 3$, for 5 it is between $1 / 3$ and 1 , and for 4 it is above 1 . All crossings for which some of the $u_{n}$ values have incorrect sign give $\Delta u_{n} /\left|\bar{u}_{n}\right|>0.9$.

Figure 3 revealed a clear tendency for $\left|u_{n}^{*}\right|$ to be larger than $\left|u_{n}\right|$. We suspect that this tendency is caused by the presence of localized substructures of the magnetopause, like undulations or magnetic islands. Tests with model magnetic fields show that MVB tends to smooth out substructures and find a normal aligned with the average gradient, whereas the $\Delta t$ of the time-lag analysis may be strongly affected by the passage of localized substructures. In general, the gradients associated with these substructures are often not aligned with the magnetopause normal but have a substantial tangential component. Since the HT velocity is also mainly tangential, its component along the gradients of the substructures may be substantially larger than $u_{n}=\mathbf{V}_{\mathrm{HT}} \cdot \mathbf{n}$. Thus values of $u_{n}^{*}$ calculated from the passage of localized substructures will typically be larger than $u_{n}$, as indeed we find them to be.

We conclude that reliable values of the magnetopause velocity can be derived by use of analysis techniques for singlespacecraft data, provided the normal vector determinations from the different techniques are consistent and have small

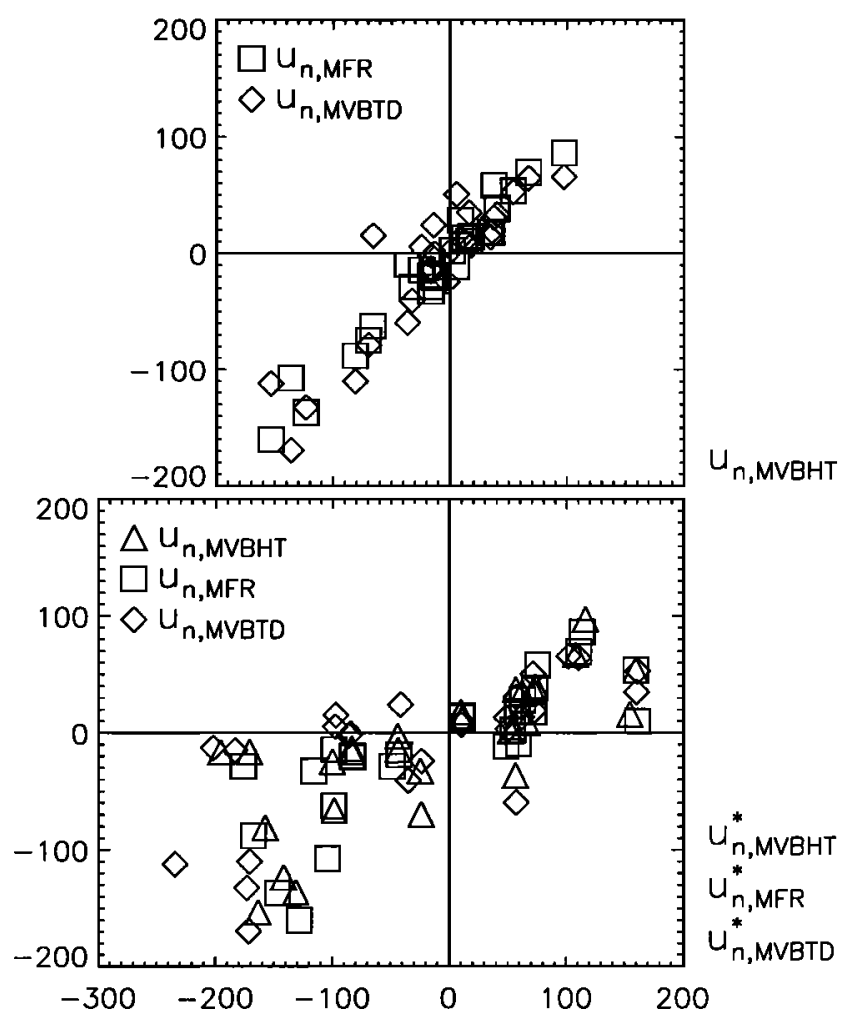

Figure 3. Scatter plots of magnetopause velocities $u_{n}$ in $\mathrm{km} / \mathrm{s}$. Lower diagram: $u_{n}$ from MVBHT, MFR, and MVBTD versus $u_{n}^{*}$, the corresponding velocities from time-lag analysis. Upper diagram: $u_{n}$ from MFR and MVBTD versus $u_{n}$ from MVBHT. 
error estimates. Based on the present investigation, we cannot decide if the velocities from single-spacecraft methods are better or worse than the velocities from the dual spacecraft time-lag technique. However, it is clear that extreme caution must be exercised in the choice of structures used in the determination of the time lag.

Acknowledgments. We thank G. Paschmann and H. Lühr for providing data. Research was performed under the auspices of the International Space Science Institute, Bern, Switzerland. Research at Dartmouth was supported by NASA under Grants NAG 5-6268 and 5-7185. We note with sadness the death of Norbert Sckopke and dedicate this paper to the memory of a selfless and skilled colleague.

\section{References}

Berchem, J. and C. T. Russell, The thickness of the magnetopause current layer, J. Geophys. Res., 87, 2108, 1982.

Cahill, L. J., and P. G. Amazeen, The boundary of the geomagnetic field, J. Geophys. Res., 68, 1835, 1963.

Fairfield, D. H., Average and unusual locations of the Earth's magnetopause and bow shock, J. Geophys. Res., 76, 6700, 1971.

Kaufmann, R. L., and A. Konradi, Explorer 12 magnetopause observations: Large-scale non-uniform motion, J. Geophys. Res., $74,3609,1969$.

Khrabrov, A. V., and B. U. Ö. Sonnerup, Orientation and motion of current layers: Minimization of the Faraday residue, Geophys. Res. Lett., 25, 2373, 1998.
Phan, T. D. and G. Paschmann, Low-latitude dayside magnetopause and boundary layer for high magnetic shear: 1. Structure and motion, J. Geophys. Res., 101, 7801, 1996.

Sonnerup, B. U. Ö., and M. Scheible, Minimum and maximum variance analysis, in Analysis Methods for Multi-Spacecraft Data, edited by G. Paschmann, and P. W. Daly, pp. 185220, Int. Space Sci. Inst., Bern, Switzerland, and Eur. Space Agency, Paris, France, 1998.

Sonnerup, B. U. Ö., I. Papamastorakis, G. Paschmann, and H. Lühr, Magnetopause properties from AMPTE/IRM observations of the convection electric field: Method development, $J$. Geophys. Res., 92, 12,137, 1987.

Terasawa, T., H. Kawano, I. Shinohara, T. Mukai, Y. Saito, M. Hoshino, A. Nishida, S. Machida, T. Nagai, T. Yamamoto, and S. Kokubun, On the determination of a moving MHD structure: Minimization of the residue of integrated Faraday's equation, J. Geomagnetism and Geoelectricity, 48, 603, 1996.

T. M. Bauer, Max-Planck-Institut für extraterrestrische Physik, Garching, Germany. (e-mail:thb@mpe.mpg.de)

M. W. Dunlop, Space and Atmospheric Physics, Imperial College, London, UK.

A. N. Fazakerley, Mullard Space Science Lab, Surrey, UK.

B. U. Ö Sonnerup and A. V. Khrabrov, Thayer School of Engineering, Dartmouth College, Hanover, NH, USA.

(Received February 14, 2000; accepted March 28, 2000.) 